\title{
ON ORDERABILITY OF TOPOLOGICAL GROUPS
}

\author{
G. RANGAN \\ The Ramanujan Institute \\ University of Madras \\ Madras-5, India \\ (Received October 15, 1984)
}

ABSTRACT. A necessary and sufficient condition for a topological group whose topology can be induced by a total order compatible with the group structure is given and such groups are called ordered or orderable topological groups. A separable totallydisconnected ordered topological group is proved to be non-archimedean metrizable while the converse is shown to be false by means of an example. A necessary and sufficient condition for a non-totally disconnected locally compact abelian group to be orderable is also given.

KEY WORDS AIND PHRASES. TOpological groups, orderable or ordered topological groups, non-archimedean metrizable, totally disconnected, locally compact abelian groups. 1980 AMS SUBJECT CLASSIFICATION CODES. 22A05, 54 F05.

\section{INTRODUCTION.}

Topological groups whose topology can be induced by a total order are called topologically orderable groups and they are studied by Nyikos and Reichel [1] and Venkataraman, Rajagopalan and Soundarajan [2]. In this paper we give a necessary and sufficient condition so that topological group is orderable in the sense that it admits a total order which induces the topology of the topological group and which is also compatible with its group structure. We call such groups ordered topological groups. As a first step to this we give a necessary and sufficient condition that a group be orderable so that it admits a totoal order compatible with the group structure and such groups are called ordered groups. Venkataraman, Rajagopalan, and Soundararajan have shown (Theorem 2.6, [2] ) that a separable totally-disconnected topological group is a topologically orderable group if and only if it is metrizable and zero dimensional. It turns out that a separable totally-disconnected ordered topological group is nonarchimedean metrizable (in the sense of Rangan [3]) while the converse fails to be true. It is interesting to note that the totally-disconnected non-archimedean metrizable locally compact abelian topological group $\left(Q_{p},+\right)$ of $p$-adic numbers under addition admits an order that is compatible with the group structure (+) alone and another order that is compatible with its topology alone but admits no order that is compatible with 
both its group structure + and sufficient condition for a non-totally disconnected locally compact abelian group to be orderable.

2. ORDERABILITY OF A TOPOLOGICAL GROUP.

THEOREM 2.1. A group $G$ can be ordered if and only if a subgroup $C(x)$ and a homomorphism $f_{x}$ from $C(x)$ into the additive group of real numbers can be found corresponding to each $x \neq e$ (e being the identity of $G$ ) which satisfies the following conditions

(i) $x \in C(x)$ and $f_{x}(x) \neq 0$ for every $x$ in $G, x \neq e$,

(ii) for $y$ in $C(x), C(y) \subseteq C(x)$

(iii) for $\mathrm{x}, \mathrm{y}$ in $\mathrm{G}, \mathrm{x} \neq \mathrm{e}, \mathrm{y} \neq \mathrm{e}$, either $\mathrm{C}(\mathrm{x})=\mathrm{C}(\mathrm{y})$ and $\mathrm{f}_{\mathrm{x}}=\mathrm{f}_{\mathrm{y}}$ or $\mathrm{C}(\mathrm{x}) \varsubsetneqq \mathrm{C}(\mathrm{y})$ in which case $\mathrm{f}_{\mathrm{y}}(\mathrm{x})=0$,

(iv) for every $a$ in $G, a C(x) a^{-1}=C(z)$ where $z=a x a^{-1}$ and $f_{x}(t)=f_{z}\left(a t a^{-1}\right)$ for every $t$ in $C(x)$ i.e. if $I_{a}: x \rightarrow a x a^{-1}$ is the inner automorphism determined by the element $a$ in $G$ then $f_{x}=f_{z}$ oI ${ }_{a}$.

Further for the order on $G, C(x)$ for each $x$ is the smallest convex subgroup containing $x$ and $C(x)=$ ker $f_{x}$ is the largest convex subgroup not containing $x$.

PROOF. Let $G$ be an ordered group. For each $x \neq e$ in $G$ let $C(x)$ denote the smallest convex subgourp containing $x$ and $C(x)^{*}$ be the largest convex subgroup not containing $x$. Then it is well known (see [4] and [5]) that $C(x)^{*}$ is a normal subgroup of $\mathrm{C}(\mathrm{x})$ and that $\mathrm{C}(\mathrm{x}) / \mathrm{C}(\mathrm{x})^{*}$ is order isomorphic to a subgroup of the reals and we identify this quotient group with the corresponding subgroup of the reals. Let $f_{x}$ be the natural map of $c(x)$ to $c(x) ! C(x)^{*}$. Then clearly $f_{x}(x) \neq 0$. Since $C(x)^{*}$ and $C(y)^{*}$ are the largest convex subgroups contained in $C(x)$ and $C(y)$ respectively, $C(x)=C(y)$ implies $C(x)^{*}=C(y)^{*}$ and hence $f_{x}=f_{y}$. If $C(x) \neq C(y)$ then being convex subgroups of an ordered group either $C(x) \subsetneq C(y)$ or $C(y) \subsetneq C(x)$. Hence (i), (ii) and (iii) of the theorem are easily verified. Inner automorphism being order preserving it follows that $\mathrm{aC}(\mathrm{x}) \mathrm{a}^{-1}=\mathrm{C}\left(\mathrm{axa} \mathrm{a}^{-1}\right)$ and $\mathrm{aC}(\mathrm{x})^{*} \mathrm{a}^{-1}=\mathrm{C}\left(\mathrm{axa}^{-1}\right)^{*} \cdot \mathrm{I}_{\mathrm{a}}$ induces an order isomorphism $\mathrm{I}_{\mathrm{a}}^{*}$ between the quotient groups $C(x) / C(x)^{*}$ and $C(z) / C(z)^{*}$ where $z=a x a^{-1}$. If we identify these two subgroups of the reals forgetting the order isomorphism between them we get that $\mathrm{f}_{\mathrm{x}}=\mathrm{f}_{\mathrm{z}} \mathrm{oI}$ a which proves (iv) of the theorem.

Conversely if G satisfies the conditions of the theorem we show that the set $P$ of all elements such that $f_{x}(x)>0$ serves as the strict positive part of an ordering on $G$. For $x \in G, C(x)=C\left(x^{-1}\right)$ by $(i i)$ and hence $f_{x}=f_{x}-1 . x \in P \Leftrightarrow f_{x}(x)>0$ $\Leftrightarrow \mathrm{f}_{\mathrm{x}}-1\left(\mathrm{x}^{-1}\right)<0 \Leftrightarrow \mathrm{x}^{-1} \notin \mathrm{P}$.

We now observe a useful property of the subgroups $C(x)$ 's which we call the property $P$. 
PROPERTY P. If $a, b \in G, a \neq e, b \neq e$ and $c(b) \subsetneq c(a)$ then $c(a)=c(a b)=$ $\mathrm{C}(\mathrm{ba})$.

For $\mathrm{C}(\mathrm{b}) \stackrel{\mathrm{f}}{\neq} \mathrm{C}(\mathrm{a}) \quad \mathrm{ab} \in \mathrm{C}(\mathrm{a})$ and $\mathrm{f}_{\mathrm{a}}(\mathrm{b})=0 \quad \mathrm{C}(\mathrm{ab}) \leq \mathrm{C}(\mathrm{a})$ and $\mathrm{f}_{\mathrm{a}}(\mathrm{b})=0$. Now $C(a b) \varsubsetneqq c(a) \Rightarrow 0=f_{a}(a b)=f_{a}(a)+f_{a}(b) \Rightarrow f_{a}(a)=0$ since $f_{a}(b)=0$, a contradiction to $(i)$. Hence $C(a)=C(a b)$. Similarly it can be seen that $C(a)=c(b a)$.

If $f_{x}(x)>0, f_{y}(y)>0$ and $C(x)=C(y)$ then $C(x y)=C(x)=C(y)$ and $f_{x y}(x y)=$ $\mathrm{f}_{\mathrm{x}}(\mathrm{x})+\mathrm{f}_{\mathrm{y}}(\mathrm{y})>0$ since $\mathrm{f}_{\mathrm{x}}=\mathrm{f}_{\mathrm{y}}=\mathrm{f}_{\mathrm{xy}}$. For, otherwise since $\mathrm{xy} \in \mathrm{C}(\mathrm{x}), \mathrm{C}(\mathrm{xy}) \subsetneq$ $C(x)=C(y)$ and hence by $(i i i), 0=f_{x}(x y)=f_{x}(x)+f_{y}(y)>0$, a contradiction. If however $\mathrm{C}(\mathrm{x}) \neq \mathrm{C}(\mathrm{y})$ let us suppose that $\mathrm{C}(\mathrm{x}) \subsetneq \mathrm{C}(\mathrm{y})$ (the case $\mathrm{C}(\mathrm{y}) \underset{\mathrm{C}}{\subsetneq}(\mathrm{x})$ can be similarly dealt with). By Property $P, C(x y)=C(y)$ and so $f_{x y}=f_{y}$ and $f_{x y}(x y)=$ $\mathrm{f}_{\mathrm{y}}(\mathrm{x})+\mathrm{f}_{\mathrm{y}}(\mathrm{y})>0$. j.e. $\mathrm{x}, \mathrm{y} \in \mathrm{P}$ implies that $\mathrm{xy} \in \mathrm{P}$.

Let $x \in P, a \in G$. Then $f_{x}(x)>0$. By (iv) of the theorem we have $a C(x) a^{-1}=$

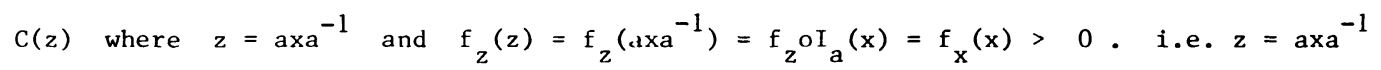
belongs to P. Now by Thenrem 2, p. 13, [4] it follows that $G$ is an ordered group.

To prove the second part of the theorem we first show that $C(x)$ is a convex subgroup by showing that $y \in C(x), e<t<y$ implies that $y t^{-1} \epsilon C(x)$ and hence $t \in C(x)$. This is done by proving that $c(x) \underset{f}{c}\left(y t^{-1}\right)$ is impossible, thereby proving that $C(x) \supseteq C\left(y^{-1}\right)$. Suppose $C(x) \varsubsetneqq C\left(y t^{-1}\right)$. From the assumption $y \in C(x)$ and condition (ii) of the theorem we get that $C\left(y^{-1}\right)=C(y) \subseteq C(x) \subsetneq C\left(y t^{-1}\right)$. Property $P$ now implies that $C\left(y t^{-1}\right)=C\left(y^{-1} y t^{-1}\right)=C\left(t^{-1}\right)=C(t)$. We will now show that this is impossible. For $\mathrm{C}(\mathrm{t})=\mathrm{C}\left(\mathrm{yt}^{-1}\right)=\mathrm{C}\left(\mathrm{ty}^{-1}\right)$ implies $\mathrm{f}_{\mathrm{t}}=\mathrm{f}_{\mathrm{yt}}-1=\mathrm{f}_{\mathrm{ty}}-1$ and so $0<$

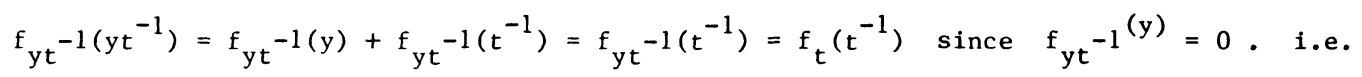
$\mathrm{f}_{\mathrm{t}}(\mathrm{t})<0$ a contradiction to the choice of $\mathrm{t}$. Thus we get that $\mathrm{C}\left(\mathrm{yt}^{-1}\right) \subseteq \mathrm{C}(\mathrm{x})$ by (iii) of the theorem. In other words $\mathrm{yt}^{-1} \in \mathrm{C}(\mathrm{x})$.

We now prove that $\mathrm{C}(\mathrm{x})$ is the smallest convex subgroup containing $\mathrm{x}$. For this let us suppose that $C$ is any conves subgroup containing $x$ and let us assume that $x>e$ and $t \in C(x), t \neq x, t>e$. This implies $C(t) \subseteq C(x)$.

CASE 1. $C(t) \varsubsetneqq C(x)$.

$C(t)=C\left(t^{-1}\right)$ and Property $P$ imply that $C(x)=C\left(x t^{-1}\right)$ and so $f_{x}=f_{x t}-1$. Now $\mathrm{f}_{x t^{-1}}\left(x t^{-1}\right)=\mathrm{f}_{x}\left(x t^{-1}\right)=\mathrm{f}_{x}(\mathrm{x})+\mathrm{f}_{\mathrm{x}}\left(\mathrm{t}^{-1}\right)=\mathrm{f}_{\mathrm{x}}(\mathrm{x})>0$ since $\mathrm{f}_{\mathrm{x}}\left(\mathrm{t}^{-1}\right)=0$ in view of $C\left(t^{-1}\right) \varsubsetneqq C(x)$. Hence $x>t>e$. The convexity of $c$ implies that $t \epsilon$. CASE 2. $C(x)=C(t)=C\left(x t^{-1}\right)$.

Then $f_{x t}-1=f_{x}=f_{t}$. We now discuss the two possibilities $f_{x}(x) \geqslant f_{x}(t)$ and $f_{x}(x)<f_{x}(t)$ separately. 
$f_{x}(x) \geqslant f_{x}(t) \Rightarrow f_{x t}-1\left(x t^{-1}\right)>0$ by $(i)$ of the Theorem since $t \neq x$. i.e. $\mathrm{e}<\mathrm{t}<\mathrm{x}$. Again from the convexity of $\mathrm{C}$ we conclude that $\mathrm{t} \epsilon \mathrm{C}$.

$$
\mathrm{f}_{\mathrm{x}}(\mathrm{x})<\mathrm{f}_{\mathrm{x}}(\mathrm{t}) \Rightarrow \mathrm{f}_{\mathrm{x}}(\mathrm{t})<\mathrm{nf}_{\mathrm{x}}(\mathrm{x}) \text { for some integer } \mathrm{n} \Rightarrow \mathrm{f}_{\mathrm{x}}\left(\mathrm{x}^{\mathrm{n}} \mathrm{t}^{-1}\right)>0 \Rightarrow \mathrm{c}\left(\mathrm{x}^{\mathrm{n}} \mathrm{t}^{-1}\right)=
$$

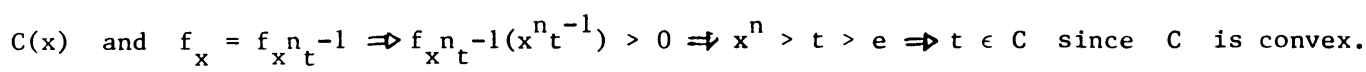
In either case $t \in C$.

CASE 3. $C\left(x t^{-1}\right) \varsubsetneqq C(x)=C(t)$. Then $f_{x}\left(x t^{-1}\right)=0$ i.e. $f_{x}(x)=f_{x}(t)$. From Property $P$ we get that $c\left(x^{2} t^{-1}\right)=c(x)$

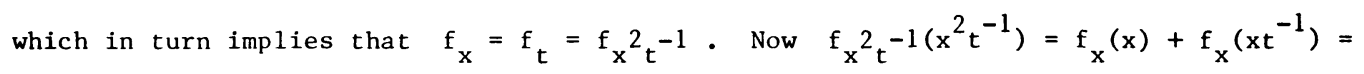
$f_{x}(x)>0$ since $f_{x}\left(x t^{-1}\right)=0$. i.e. $x^{2}>t>e$. The convexity of $c$ now implies that $t \in C$.

Thus we see that in all cases $C(x) \subseteq C$. For $x<e \quad C \supseteq C\left(x^{-1}\right)=C(x)$ which proves that $C(x)$ is the smallest convex subgroup containing $x$.

$f_{x}$ is an order preserving homomorphism from $c(x)$ to the reals. For let $z, y \in C(x)$ and $z>y \cdot$ Then $C\left(z y^{-1}\right) \subseteq C(x) \cdot C\left(z y^{-1}\right) \subsetneq C(x) \Rightarrow f_{x}\left(z y^{-1}\right)=0$ $\mathrm{f}_{\mathrm{x}}(\mathrm{z})=\mathrm{f}_{\mathrm{x}}(\mathrm{y}) \cdot \mathrm{C}\left(\mathrm{zy}^{-1}\right)=\mathrm{C}(\mathrm{x}) \Rightarrow \mathrm{f}_{\mathrm{x}}=\mathrm{f}_{\mathrm{zy}}-1 \Rightarrow 0<\mathrm{f}_{\mathrm{zy}}-1\left(\mathrm{zy}^{-1}\right)=\mathrm{f}_{\mathrm{x}}\left(\mathrm{zy}^{-1}\right)=\mathrm{f}_{\mathrm{x}}(\mathrm{z})-$ $f_{x}(y) \Rightarrow f_{x}(y)<f_{x}(z)$. In either case $f_{x}(z) \geq f_{x}(y)$. This proves that $\operatorname{Ker} f_{x}=$ $C(x)^{*}$ is a convex subgroup of $C(x)$ and hence also of $G$.

It remains for us to show that $C(x)^{*}$ is the largest convex subgroup not containing $x$. For this let us suppose that $C$ is any convex subgroup not containing $x$. Let $t \in C$. Then since $x \notin C, C \subseteq C(x)$ and so $t \in C(x)$. If $f_{x}(t) \neq 0$ then $C(t)=C(x)$ by (i) of the theorem and $C(t)$ being the smallest convex subgroup containing $t, C(x)=C(t) \subseteq C$. i.e. $x \in C$ a contradiction. Hence $f_{x}(t)=0$. i.e. $t \in \operatorname{Ker} \mathrm{f}_{\mathrm{x}}=\mathrm{C}(\mathrm{x})^{*}$. This completes the proof of the theorem.

Even though possibly one can prove Theorem 2.1 in a different way using Theorem 11 , p.51 of [4] or Theorem 2 of [5] we have prefered the above proof since it is elementary. The above form of formulating the theorem gives rise in a natural way for a criterion of orderability of a topological group as well.

REMARK. $C$, the intersection of all $C(x), x \in G, x \neq e$ is the first convex subgroup of $G$. Hence when $C=(e)$ the above theorem gives a necessary and sufficient condition that $G$ may be made into an ordered group without first convex subgroup.

THEOREM 2.2. A non-discrete topological group $G$ can be ordered so that the interval topology of the order coincides with the given topology and also simultaneously this order is compatible with the group structure of $G$ if and only if $G$ satisfies the following criterion (v) in addition to those of Theorem 2.1.

(v) either the intersection of all the $C(x), x \in G, x \neq e$ is the singleton (e) in which case the collection $\{C(x)\}, x \in G$ form a neighbourhord base at the 
identity $e$ of $G$ or the intersection $c$ of all the $C(x), x \in G, x \neq e$ is an open subgroup of $G$ and for each $x$ in $C, f_{x}$ is an open continuous homomorphism into the reals.

PROOF. In view of Theorem 2.1, it is enough to show that condition ( $v$ ) above is equivalent to the coincidence of the interval topology and the given topology of the group $\mathrm{G}$.

Let now $G$ be an ordered group. $G$ with the interval topology is a topological group (see $4.19[6]$ ). We define $C(x)$ and $C(x)^{*}$ for $x \in G, x \neq e$ as in Theorem 2.1 . CASE 1. $\mathrm{nC}(\mathrm{x})=(\mathrm{e})$.

Let $I$ be an open interval containing $e$. Then since $G$ is not discrete there exists a $y>e$ such that $y$ and $y^{-1}$ are in $I$. Since $n C(x)=(e)$, there exists $x \in C$ such that $y \notin C(x)$. $t>e, t \in C(x)$ implies $t \leq y$ since $C(x)$ is convex. Hence $t \in I$ i.e. $C(x) \subseteq I$ and this proves that $C(x), x \in G$ form a neighbourhood base of e in $G$.

CASE 2. $n C(x)=C \neq(e)$.

Then $C$ is a convex subgroup and hence open in $G$. For $x \in C$ it is clear that $C=C(x)$. Hence for $x, y \in C, C=C(x)=C(y)$ and so $f_{x}=f_{y}=f$ (say). $x \neq y$, $\mathrm{x}, \mathrm{y} \in \mathrm{C} \Rightarrow \mathrm{f}=\mathrm{f}_{\mathrm{x}}=\mathrm{f}_{\mathrm{y}}=\mathrm{f}_{\mathrm{xy}}-1 \Rightarrow 0 \neq \mathrm{f}_{\mathrm{xy}}-\mathrm{l}\left(\mathrm{xy}^{-1}\right)=\mathrm{f}(\mathrm{x})-\mathrm{f}(\mathrm{y}) \Rightarrow \mathrm{f}(\mathrm{x}) \neq \mathrm{f}(\mathrm{y})$. i.e. $\mathrm{f}$ is one-to-one on $\mathrm{C} \cdot \mathrm{f}_{\mathrm{x}}$ being the cannonical map from $\mathrm{C}(\mathrm{x})$ to $\mathrm{C}(\mathrm{x}) / \mathrm{C}(\mathrm{x})$ * and $C(x) / C(x)^{*}$ being order isomorphic to a subgroup of the reals, $f_{x}$ is an open continuous homomorphism from $\mathrm{C}(\mathrm{x})$ to the reals.

To prove the converse also we consider two cases.

CASE 1. $n C(x)=(e)$.

From Case 1 of the necessity part it follows that $\{c(x)\}, x \in G, x \neq e$, form a neighbourhood base for the interval topology. By hypothesis $\{C(x)\}, x \in G, x \neq e$ form a neighbourhood base at $e$ for the given topology and hence the two topologies coincide. CASE 2. $n C(x)=C \neq(e)$.

For $x, y \in C$, it is clear from the convexity of $C(x)$ and (ii) of Theorem 2.1 that $\mathrm{f}_{\mathrm{x}}=\mathrm{f}_{\mathrm{y}}=\mathrm{f}$ (say). Then $\mathrm{f}$ is a one-to-one open continuous homomorphism from $\mathrm{C}$ to the reals, the topology of $C$ being the relative topology got from the order topology of $G$. Hence $C$ with respect to this (order) topology is homeomorphic to a subgroup of the reals with respect to its relative topology. But by hypothesis $f$ is an open continuous homomorphism from $\mathrm{C}$, taken with the relative topology got from the given topology on $G$ onto this subgroup of the reals. Now $f$ is one-to-one implies that the two topologies on $\mathrm{C}$ conincide. C being an open subgroup in both the topologies on $G$, the rwo topologies on $G$ themselves coincide.

COROLLARY 2.3. Let $G$ be an ordered topological group (i.e. the topology of $G$ is giben by a total order compatible with the group structure of $G$ ). Then the component of the identity $e$ of $G$ is either the trivial subgroup (e) or it is an open subgroup topologically isomorphic to the additive group of reals. 
PROOF. If $n C(x)=(e)$ by Theorem 2.2 above $G$ becomes a zero dimensional group. If $n C(x)=C \neq(e)$ then $C$ is the component of identity. For, each $C(x)$ being an open and closed subgroup, contains the component of $e$ and so $C$ itself contains the component. But the component being a connected subgroup is also a convex subgroup (see Proposition $1.3(2),[2]$ ) and hence contains $C$ which proves that $C$ is the same as the component. From Theorem 2.2 it follows easily that $C$ is topologically isomorphic to the additive group of reals.

3. TOTALLY DISCONNECTED ORDERED GROUPS.

A metric $d$ on a set $X$ is said to be a non-archimedean metric if $d$ satisfies the stronger triangle inequality

$$
d(x, y) \leqslant \max (d(x, z), d(z, y))
$$

for $\mathrm{x}, \mathrm{y}, \mathrm{z} \in \mathrm{X}$. A topological group is said to be non-archimedean metrizable if there exists a right (or left) invariant metric on $G$ which induces the topology of $G$ (see Rangan [3]).

LEMMA 3.1. Suppose a topological group $G$ is such that its topology is given by a non-archimedean metric $d$ then there is an equivalent non-archimedean right (or left) invariant metric $\rho$ on $G$ (i.e. a non-archimedean metric $\rho$ which gives the same topology as d).

PROOF. When $d$ is a non-archimedean metric on $G$ then the metric $d^{\prime}=d^{\prime} / 1+d$ also defines the same topology on $G$. Put $\rho(x, y)=\sup d^{\prime}(x z, y z)$ where supremum is taken as $z$ varies in $G$. Then $\rho$ is well-defined since $d^{\prime}$ is bounded. It is easy to check that $\rho$ is a right invariant non-archimedean metric on $G$. We will now show that the topologies induced by $\rho$ and $d$ ' coincide.

Since $\rho^{\prime}(x, y)>d^{\prime}(x, y)$ for all $x, y \in G$, it is clear that $V_{n} \subset U_{n}$ for all positive integers $n$ where $V_{n}=\{x: \rho(x, e)<1 / n\}$ and $U_{n}=\left\{x: d^{\prime}(x, e)<1 / n\right\}$. i.e. the topology induced by $\rho$ is finer than the topology induced by d'. To prove that the two topologies are the same we will show that each $V_{M}$ contains a $U_{k}$ for some $k$. Corresponding to the Integer $M>0$, choose positive integers $n, m$ such that

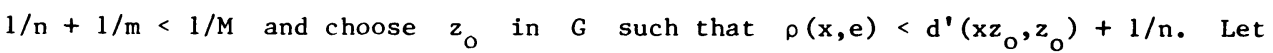
$W=\left\{x: d^{\prime}\left(x z_{0}, z_{0}\right)<1 / m\right\}$. Then $W z_{0}$ is the open sphere with centre $z_{0}$ and radius $1 / m$ with respect to the metric $d^{\prime}$. The continuity of $x \rightarrow x z$ at $x=e$ implies the existence of a positive integer $k$ such that $U_{k_{0}} z_{0} W z_{0}$. Let $x \in U_{k}$. Clearly $x$ is in $W$. Hence $\rho(x, e)<d^{\prime}\left(x z_{0}, z_{o}\right)+1 / n<1 / m+1 / n<1 / M$ proving thereby that $\mathrm{U}_{\mathrm{k}} \subset \mathrm{V}_{\mathrm{M}}$. This completes the proof of the lemma.

THEOREM 3.2. Let $G$ be a separable totally disconnected ordered topological group. Then $G$ is non-archimedean metrizable.

PROOF. By Theorem 2.6 of [2], it follows that $G$ is topologically orderable, metrizable, zero-dimensional group and so carries a non-archimedean metric inducing the topology of G (see proof of Theorem 7 of [1] or [7]). Now from Lemma 3.1 it follows that $G$ is non-archimedean metrizable. 
Venkataraman, Rajagopalan and Soundararajan proved (see Theorem 2.6, [2]) that a separable totally disconnected group is topologically orderable (i.e. the topology is given by a total order) if and only if it is metrizable and zero-dimensional. Nonarchimedean metrizable groups are totally disconnected groups (see Rangan [3]). Hence in view of Theorem 3.2 above it is natural to ask whether the converse of Theorem 3.2 is also true. i.e. Is it possible to total order a non-archimedean separable topological group in such a way that the order is compatible with both the group structure and the topology? That the answer to this question is in the negative is shown by Theorem 3.4 given below. For the counter example given in Theorem 3.4 we require the following definition.

Let $Q$ denote the field of rational numbers. Let $p$ be a fixed prime number. Each $x$ in $Q$ can be written uniquely in the form $p^{s} x$ ' where $p$ does not divide the numerator and denominator of $x^{\prime}$ and $s$ is an integer. We define $|x|_{p}=$ $1 / p^{s}$ and $d(x, y)=|x-y| p$ for $x, y$ in $Q$. Then $d$ is easily seen to be a nonarchimedean metric. The completion of $Q_{p}$ of $Q$ with this metric (which can be made into a field extending the addition and multiplication in $Q$ ) is called the field of p-adic numbers. We consider the additive group of this field $Q_{p}$ and denote it by $Q_{p}$ itself. It is a separable totally disconnected group (see section 2, [8] for details).

LEMMA 3.3. (see van Rooij [8]). In $Q_{p}$ the series $\sum n . n$ ! converges to -1 . PROOF. Since $|n !|_{p}$ and $|n \cdot n !|_{p}$ tend to zero as $n$ tends to infinity, it follows that $\sum n !=A$ and $\sum n \cdot n !=B$. Then $A+B=\sum n !+\sum n \cdot n !=\sum(n+1) !=A-1$ which proves that $B=-1$.

THEOREM 3.4. There is no order $>$ on $\left(Q_{p},+\right)$ which is compatible with both + and the usual topology of $Q_{p}$ even though it admits an order compatible with the topology of $Q_{p}$ alone and an order compatible with the group structure + alone.

PROOF. Suppose $Q_{p}$ admits a total order compatible with the group structure and topology. Let $1>0$ under this order. From the compatibility of the group structure and order it follows that $s_{n}=\sum_{k=1}^{n} k \cdot k !>0$ and from the compatibijity of the order and topology it lollows by Lemma 3.3 that $-1=1$ im $s_{n}>0$ which is a contradiction. Similarly $1<0$ is also impossible. This proves that $Q_{p}$ admits no order compatible simultaneously with the group structure + and the topology. However by Theorem 2.6 of [2] it follows that it admits an order which induces the topology of $Q_{p}$ (see also Remark 5.7 of [2]). Since $Q_{p}$ is a torsion-free abelian group it admits a total order compatible with the group structure + alone (Corollary 5, p.36, [4]).

4. NON-TOTALLY DISCONNECTED ORDERED GROUPS.

In this section we consider only abelian non-totally disconnected groups. In what follows we refer the reader to Wright [9] for the definitions of radical-free, maximal 
radical free etc.

THEOREM 4.1. A non-totally disconnected locally compact ableian topological group is orderable if and only if it is maximally radical free and its component is an open subgroup of $G$ topologically isomorphic to the reals.

PROOF. Suppose $G$ is a non-totally disconnected locally compact abelian group whose topology is given by a total order which is compatible with the group structure of G. Then by Corollary 2.3 its component $\mathrm{C}$ is an open subgroup topologically isomorphic with the reals. Now by Theorem 5.1 of Wright [9] it follows that $G$ is maximally radical-free in its interval topology.

Let now $G$ satisfy the conditions of the Theorem. If $G$ is connected then by Theorem 5.2 of Wright [9] $G$ is topologically isomorphic to the reals and so $G$ is orderable. If not since the component of the identity is open and topologically isomorphic to the reals $G$ is one-dimensional, $G / C$ is discrete and maximally radical free by Theorem 5.3 of Wright [9] and hence torsion-free by Theorem 4.1 of Wright [9]. By Theorem 1 of Isiwata [10], G can be totally ordered so that the order topology coincides with the given topology of $G$ and this order is compatible with the group structure as well.

ACKNOWLEDGEMENT. I thank Professors M. Rajagopalan and R. Venkataman for the many useful discussions I had with them while preparing this paper.

\section{REFERENCES}

1. NYIKOS, P.J., and REICHEL, H.C. Topologically orderable groups, Gen. Topology Appl. $\underline{5}(1975), 195-204$.

2. VENKATARAMAN, M., RAJAGOPAlAN, M., and SOUNDARAJAN, T. Orderable topological spaces, Gen. Topology App1. 2 (1972), 1-10.

3. RANGAN, G. Non-archimedean metrizability of topological groups, Fund. Math. 5 (1970), 179-182.

4. FUCHS, L. Partially ordered algebraic systems, Pergaman Press (1963).

5. IWASAWA, K. On linearly ordered groups, J. Math. Soc. Japan 1 (1948), 1-9.

6. HEWITT, E. and ROSS, K. A. Abstract Harmonic Analysis, vol. I, Springer-verlag (1963).

7. GROOT de, J. Non-archimedean metrics in topology, Proc. Amer. Math. Soc. $\underline{7}(1956)$,

8. ROOIJ van, A. C. $M$ and SCHIKHOF, W. H. Non-archimedean analysis, Nieuw Arch. Wisk. (3) $19(1971), 120-160$.

9. WRIGHT, F. B. Topological abelian groups, Amer. J. Math. 59(1957), 477-496.

10. ISIWATA, T. Linearisation of topological groups and ordered rings, Kodai Math. Sem. Reports (1952), 33-35. 


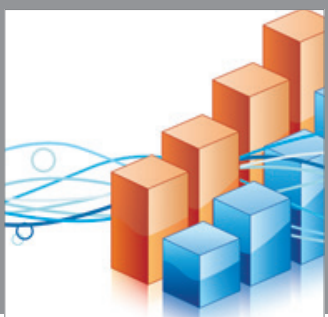

Advances in

Operations Research

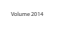

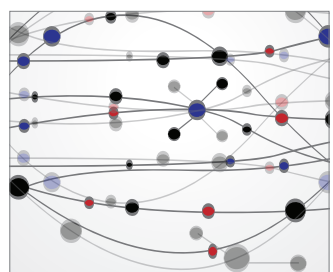

\section{The Scientific} World Journal
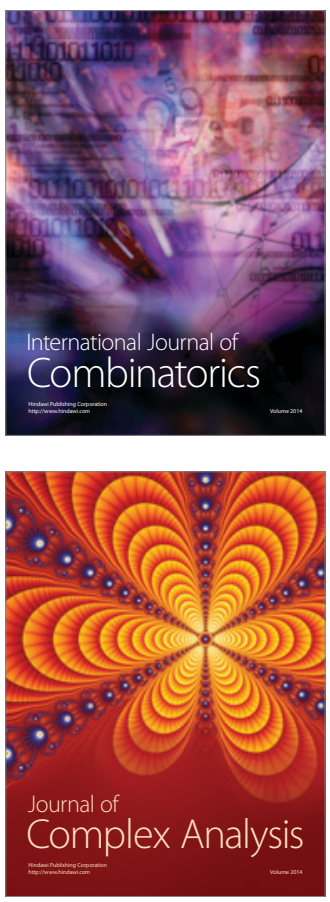

International Journal of

Mathematics and

Mathematical

Sciences
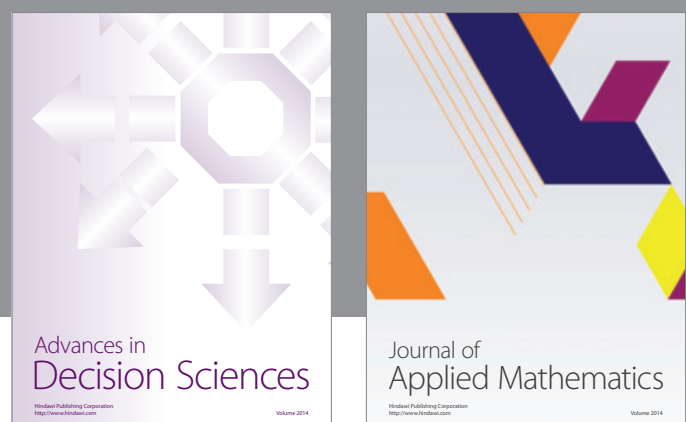

Journal of

Applied Mathematics
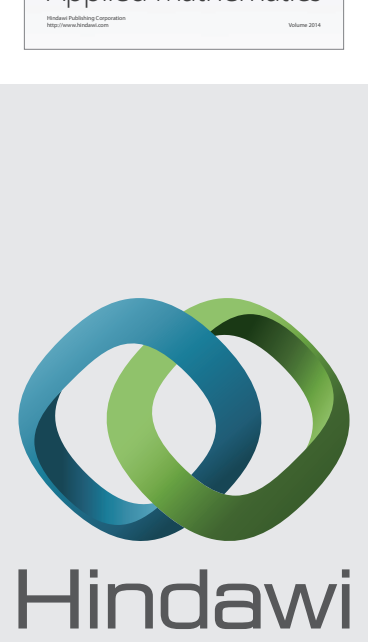

Submit your manuscripts at http://www.hindawi.com
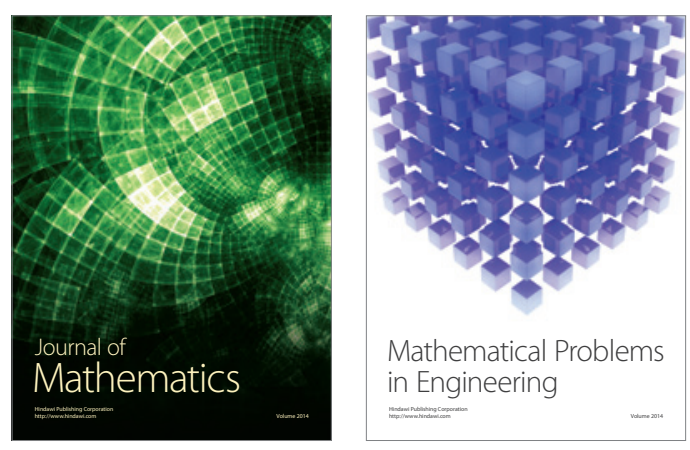

Mathematical Problems in Engineering
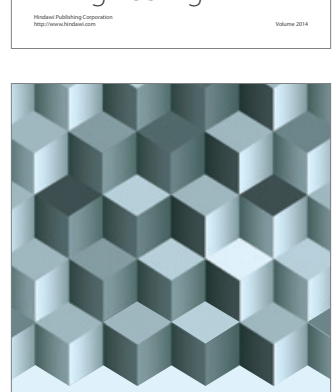

Journal of

Function Spaces
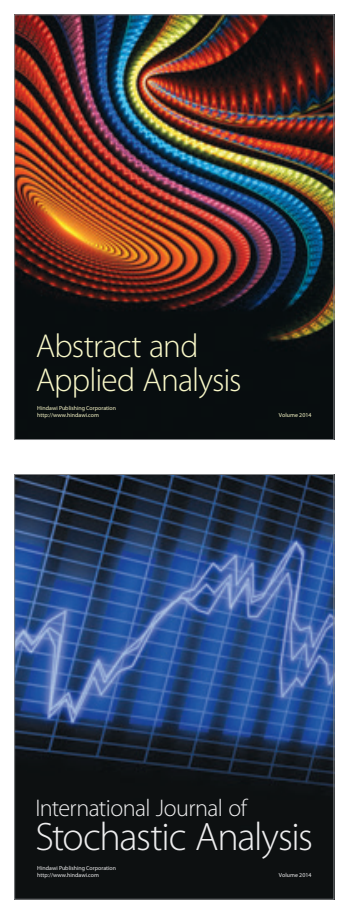

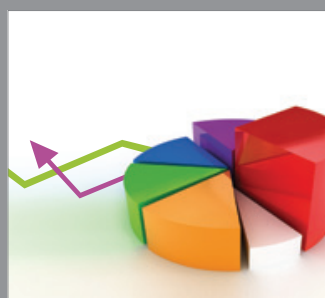

ournal of

Probability and Statistics

Promensencen
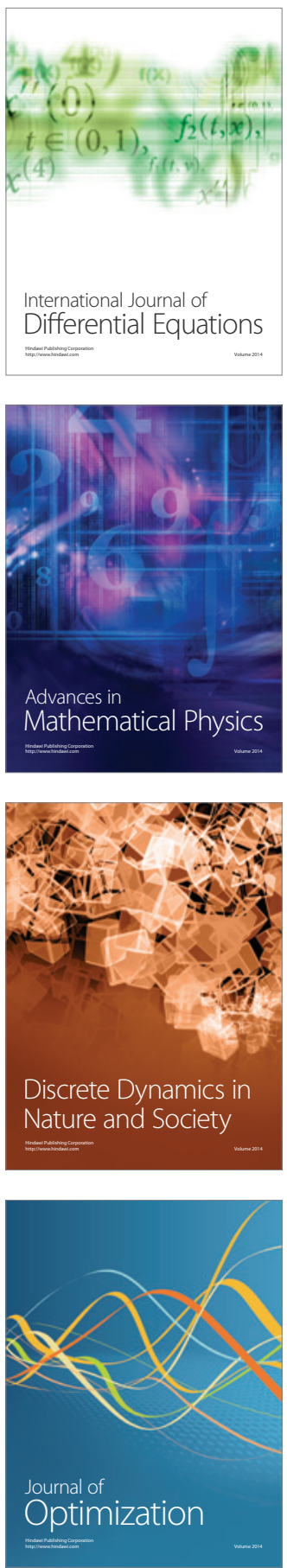